\title{
МОДЕЛЬ ОЦЕНКИ ЭФФЕКТИВНОСТИ МЕТОДОВ УСТРАНЕНИЯ РИСКОВ ФУНКЦИОНИРОВАНИЯ СЛОЖНЫХ СИСТЕМЫ
}

\author{
А. П. Алексеев, Г. В. Абрамов, И. Н. Булгакова \\ Воронежский государственный университет
}

Поступила в редакцию 25.02.2019 г.

\begin{abstract}
Аннотация. В данной статье предложена модель определения расчетно-аналитических риск-показателей, способных выступать в роли интегральных оценок рисковых событий, которые потенциально могут серьезно повлиять на эффективность системы. В модели учитывается множество критериев оценки внешних и внутренних факторов вероятных рисковых событий в системе, что позволяет использовать ее для решения многокритериальных задач определения рисков. Также данная модель реализует алгоритм оценки трудности минимизации рисков с учетом многокритериальности, используя показатели типа «трудность достижения цели». Предлагаемые оценки являются безразмерными показателями, что позволяет применять их в системах различных типов. Полученные данные могут использоваться при принятии решений о допустимости рисков, при выборе между потенциальными мерами по снижению или устранению рисков.

Ключевые слова: риск-показатели, функционирование системы, трудность достижения цели.

Annotation. The article proposes a model for determining of the analytical risk indicators, that can serve as integral assessments of risk events that can potentially seriously affect the effectiveness of the system. This model considers many criteria for evaluating external and internal factors of probable risk events and can be used for the solving of multi-criteria tasks of risk definition. It also realizes an algorithm for evaluating the difficulty of risk minimization, considering multi-criteria, using parameters of "difficult of achieving the goal". The proposed assessments are dimensionless indicators, so they can be used in systems of various types. The obtained data can be used by making decisions about the acceptability of risks, when choosing between potential measures to reduce or eliminate risks.
\end{abstract}

Keywords: risk indicators, system functioning, difficult of achieving the goal.

\section{ВВЕДЕНИЕ}

Анализ рисков представляет собой процесс, цель которого - определение тяжести и вероятности наступления неблагоприятных последствий для любой сложной системы [1]. Различные рисковые события потенциально могут серьезно повлиять на эффективность системы - свойство системы выполнять поставленную цель в заданных условиях использования и с определенным качеством. Поэтому результаты анализа используются при принятии решений о допустимости рисков, при выборе между потенциальными ме2019 рами по снижению или устранению рисков. При несоответствующем анализе рисков принимается неверное решение о дальнейших действиях по отношению к выявленным рискам, что приводит к значительным последствиям для функционирования системы [2].

Оценка риска должна быть, во-первых, не спонтанным процессом, а результатом спланированной, целенаправленной деятельности; во-вторых, должна иметь количественное выражение, поскольку деятельность по снижению риска требует мониторинга и оценки эффективности [3].

Следовательно, процедура оценки рисков и эффективности их снижения должна отвечать следующим требованиям [3]: 
1) Полученные оценки должны быть в количественном виде;

2) Процедура должна быть простой и информативной;

3) Она должна удовлетворять требованию воспроизводимости полученных оценок.

Хотя существует большое количество методов анализа рисков для различных систем $[1,3,4]$, большинству из них присущи такие недостатки, как зависимость от конкретных объектов, спорность экспертных оценок, сконцентрированность на оценке конкретных рисковых событий, ввиду чего сложно охватить широкий диапазон факторов и построить общие интегральные оценки рисков. Разработка новых методов анализа рисков остается актуальной задачей, в особенности в том, что касается целевого подхода к оценке рисков.

Предлагаемый ниже метод оценки эффективности устранения рисков нарушения функционирования системы использует безразмерные величины трудности достижения цели в качестве специальных риск-показателей, что позволяет применять его для широкого круга задач анализа рисков. Гибкость и универсальность полученных оценок позволяет сводить вопрос к известным задачам с отлаженными алгоритмами решения, в частности, к многокритериальной задача о назначениях [5].

\section{МОДЕЛЬ ОПРЕДЕЛЕНИЯ МНОГОКРИТЕРИАЛЬНЫХ РАСЧЕТНО-АНАЛИТИЧЕСКИХ РИСК-ПОКАЗАТЕЛЕЙ}

Рассмотрим некую систему, для которой существует ряд рисковых событий, каждое из которых может сказаться на эффективности функционирования этой системы. Пусть имеется $M$ таких событий, $j=1, \ldots, m$. Для устранения или хотя бы уменьшения этих рисков существует $N$ методов, $i=1, \ldots, n$, причем эффективность применения различных методов к разным рискам отличается. Критериев эффективности может несколько, $k=1, \ldots, l$.

Отметим, что каждое рисковое событие характеризуется рядом параметров, в том числе вероятностью наступления, тяжестью последствий, временем наступления и т.д. Применение методов устранения рисков приводит к тому, что эти параметры (либо часть из них) улучшаются, то есть становятся более приемлемыми для функционирующей системы. Поэтому логично в качестве критериев эффективности метода использовать величины воздействия методов на параметры рискового события (что, однако, не исключает возможности ввести дополнительные критерии).

Для оценки эффективности этих критериев используем $L$ матриц параметров:

$Q^{k}=\left(q_{i j}^{k}\right)$ - матрица эффективности $i$-го метода против $j$-го рискового события по $k$-му критерию эффективности.

По каждому критерию эффективности существуют ограничения. Если в результате применения метода параметры рискового события стали хуже, чем до применения, то этот метод не удовлетворяет минимальным требованиям. Обозначим эти требования следующим образом:

$Q m_{j}^{k}$ - минимальная эффективность метода по $k$-му критерию, необходимая для устранения $j$-го рискового события. В качестве этих требований рекомендуется выбирать текущие (до воздействия) параметры риска.

Таким образом, перед нами встает задача распределения различных методов по рисковым события, т. е. предполагается, что каждый метод применяется против одного конкретного риска.

Введем параметр $x_{i j}$, который служит для обозначения, какой метод применяется для какого рискового события.

$x_{i j}=0$, если $i$-й метод не применяется для воздействия на $j$-е рисковое событие.

$x_{i j}=1$, если $i$-й метод применяется для воздействия на $j$-е рисковое событие.

Очевидно, что должны выполняться следующие условия:

$$
\begin{gathered}
\sum_{i=1}^{n} x_{i j}=1, j=\overline{1, m} \\
\sum_{j=1}^{m} x_{i j}=1, i=\overline{1, n}
\end{gathered}
$$

Необходимо решить задачу нахождения матрицы распределения методов по рискам: 
Модель оиенки эффективности методов устранения рисков функиионирования сложных систем

\begin{tabular}{c|cccc:c} 
& 1 & 2 & $\ldots$ & $m$ & $j$ \\
\hline 1 & 0 & 0 & $\ldots$ & 1 & \\
2 & 1 & 0 & & & \\
$\ldots$ & $\ldots$ & & $x_{i j}$ & & \\
$n$ & 0 & 1 & & $x_{n m}$ & \\
\hdashline$i$ & & & & &
\end{tabular}

При следующих ограничениях:

$$
\left\{\begin{array}{l}
\sum_{i=1}^{n} x_{i j}=1, j=\overline{1, m} \\
\sum_{j=1}^{m} x_{i j}=1, i=\overline{1, n} \\
\sum_{i=1}^{n} q_{i j}^{k} x_{i j} \geq Q m_{j}^{k}, j=\overline{1, m}, k=\overline{1, l} \\
x_{i j}=\left\{\begin{array}{l}
0 \\
1
\end{array}, i=\overline{1, n}, j=\overline{1, m}\right.
\end{array}\right.
$$

Поставленная задача обычно называется «многокритериальной задачей о назначениях». Существует несколько основных методов решения задач многокритериального выбора [6], их можно разделить на четыре группы:

1. Оптимизация одного критерия, а остальные критерии выступают в роли дополнительных ограничений;

2. Ранжирование множества критериев и последовательная оптимизация по каждому из них.

3. Минимизация максимальных отклонений от наилучших значений по всем критериям;

4. Сведение многих критериев к одному путем введения весовых коэффициентов важности для каждого критерия и нормализации векторного критерия.

В нашем случае значения критериев имеют различные единицы измерения, поэтому нужно привести их к безразмерному виду, однако оценка риска нарушения функционирования системы должна быть наглядной и легко интерпретируемой. Для одновременного учета всех критериев эффективности устранения всех рисковых событий необходимо создать интегральные риск-показатели, поэтому мы воспользуемся оценками трудности достижения цели и сведем задачу к качественным показателям. Единый риск-показатель, характеризующий рисковые события до и после применения методов устранения рисков, может быть получен применением свертки с учетом важности каждого критерия.

Итак, дадим оценку качества и требования к качеству показателей эффективности методов устранения рисков. Сначала введем качественные показатели по каждому критерию эффективности:

$$
\begin{gathered}
\mu_{i j}^{k}=\frac{q_{i j}^{k}-\underline{Q^{k}}}{\overline{Q^{k}}-\underline{Q^{k}}}, \\
\overline{Q^{k}}=\max _{\substack{1 \leq i \leq n \\
1 \leq j \leq m}} q_{i j}^{k}, \quad \underline{Q}^{k}=\min _{\substack{1 \leq i \leq n \\
1 \leq j \leq m}} q_{i j}^{k} .
\end{gathered}
$$

где

В итоге получим $L$ матриц качественных показателей эффективности.

Параметры требования к качеству имеют вид:

$$
\varepsilon_{j}^{k}=\frac{Q m_{j}^{k}-\underline{Q}^{k}}{\overline{Q^{k}}-\underline{Q^{k}}} .
$$

В конечном итоге, получим $L$ векторов требований к качеству устранения рисков.

Необходимо отметить, что оба вида параметров $\mu$ и $\varepsilon$ измеряются в интервале $[0,1]$, причем $\varepsilon_{j} \leq \mu_{i j} \forall i$ для любого критерия эффективности. Комбинации, для которых это условие не выполняется, не удовлетворяют минимальным требованиям качества. Показатель трудности в этом случае следует приравнять единице. В остальных случаях показатель трудности будет равен:

$$
d_{i j}^{k}=\frac{\varepsilon_{j}^{k}\left(1-\mu_{i j}^{k}\right)}{\mu_{i j}^{k}\left(1-\varepsilon_{j}^{k}\right)} .
$$

На данном этапе имеется L матриц трудностей, которые необходимо свести к одной путем свертки. Введем показатели $v^{k}-$ коэффициент важности $k$-го критерия, причем:

$$
\sum_{k=1}^{l} v^{k}=1
$$

Воспользовавшись формулой сложения трудностей, получим интегральную матрицу трудностей [7]:

$$
S d_{i j}=\sum_{k=1}^{l} v^{k} d_{i j}^{k}=1-\prod_{k=1}^{l}\left(1-d_{i j}^{k}\right)^{v^{k}} .
$$


Таким образом, матрица $S d$ является матрицей интегральных риск-показателей трудности [8]. Чтобы задача могла быть решена методами задачи о назначениях, прологарифмируем оценки трудностей и обозначим их параметром:

$$
C d_{i j}=\ln \left(\frac{1}{1-S d_{i j}}\right) .
$$

Теперь запишем модель задачи поиска минимальной трудности устранения рисков функционированию системы:

$$
\left\{\begin{array}{l}
\sum_{i=1}^{n} \sum_{j=1}^{m} C d_{i j} x_{i j} \rightarrow \min \\
\sum_{i=1}^{n} x_{i j}=1, j=\overline{1, m} \\
\sum_{j=1}^{m} x_{i j}=1, i=\overline{1, n} \\
\sum_{i=1}^{n} q_{i j}^{k} x_{i j} \geq Q m_{j}^{k}, j=\overline{1, m}, k=\overline{1, l} \\
x_{i j}=\left\{\begin{array}{l}
0 \\
1
\end{array}, i=\overline{1, n}, j=\overline{1, m}\right.
\end{array}\right.
$$

Полученная нами матрица трудностей отражает интегральную оценку эффективности применения методов устранение рисков к рисковым событиям. Покажем, что величины $S d_{i j}$ можно трактовать как вероятностные характеристики $[9,10]$. Пусть $A_{j}$ - событие, состоящее в том, что не выполнены требования к качеству устранения $j$-го риска, а $B_{i}-$ событие, состоящее в том, что не выполнены требования к качеству $i$-го метода. Тогда условная вероятность $P\left(A_{j} / \overline{B_{i}}\right)$ может быть выражена формулой:

$$
\begin{gathered}
P\left(A_{j} / \overline{B_{i}}\right)=\left(1-P\left(B_{i} / A_{j}\right) P\left(B_{i}\right)\right) / \\
\left(\left(1-P\left(B_{i}\right)\right) P\left(B_{i} / A_{j}\right)\right) .
\end{gathered}
$$

Если принять $S d_{i j}=P\left(A_{j} / \overline{B_{i}}\right), \quad \mu_{i j}=$ $=P\left(B_{i} / A_{j}\right), \varepsilon_{j}=P\left(B_{i}\right)$, то формула вероятности запишется в виде ранее данной формулы трудности. Воспользуемся формулой сложения трудностей еще раз и получим вероятностную оценку:

$$
p=\sum_{i=1}^{n} \sum_{j=1}^{m} S d_{i j} x_{i j} .
$$

Этот параметр можно трактовать как интегральную оценку эффективности устранения рисков функционирования системы, а также как ожидаемую вероятность того, что последствия рисковых событий нарушат функционирование системы [7]. Высокая трудность соответствует высокой вероятности возникновения системных потерь даже в случае использования специальных методов устранения рисков, в то время как небольшая трудность показывает, что используемые методы достаточно эффективны, чтобы свести вероятность серьезного нарушения функционирования к минимуму.

\section{ЭКСПЕРИМЕНТАЛЬНЫЙ РАСЧЕТ}

Приведем пример оценки устранения рисков в системе управления предприятием, используя экспертные оценки параметров нескольких рисковых событий, а именно - вероятности наступления рисковых событий, ожидаемое время устранения последствий риска и ожидаемые затраты на устранения последствий. Данные находятся в табл. 1.

Экспертами предложено пять методов воздействия на рисковые события с целью уменьшения их показателей, эти методы характеризуются параметрами:

$Q^{1}$ - снижение вероятности наступление рискового события (т.е. новая вероятность этого события).

$Q^{2}$ - время, необходимое для реализации метода воздействия на риск (в днях).

$Q^{3}$ - издержки, связанные с реализацией метода воздействия на риск (в т. р.).

Поскольку данные из табл. 1 отражают ожидания до применения конкретных методов, используем их в качестве минимальных требований к трем параметрам, характеризующим методы устранения угроз, т.е. определим векторы $Q m_{j}^{1}, Q m_{j}^{2}$ и $Q m_{j}^{3}$ соответственно. Соотношения методов и угроз по всем трем параметрам даны в табл. 2-4.

Также введем коэффициенты важности $v_{q}^{k}: v_{q}^{1}=0,5 \quad v_{q}^{2}=0,25 \quad v_{q}^{3}=0,25$.

На основе матриц эффективности построим матрицы показателей качества: 
Модель оценки эффективности методов устранения рисков функиионирования сложньх систем

Таблица 1

Идентификация факторов риска в системе управления предприятием и их основные параметры

\begin{tabular}{|c|l|c|c|c|}
\hline № & \multicolumn{1}{|c|}{ Тип риска } & $\begin{array}{c}\text { Вероятность } \\
\text { наступления } \\
\text { события }\end{array}$ & $\begin{array}{c}\text { Ожидаемое } \\
\text { время } \\
\text { в днях }\end{array}$ & $\begin{array}{c}\text { Ожидаемые } \\
\text { затраты } \\
\text { в т. p. }\end{array}$ \\
\hline 1 & Износ основных средств & 0,48 & 200 & 600 \\
\hline 2 & $\begin{array}{l}\text { Отсутствие нужной квалификации у персо- } \\
\text { нала, недостаточная его численность }\end{array}$ & 0,42 & 180 & 300 \\
\hline 3 & $\begin{array}{l}\text { Отсутствие ритмичного графика поставок } \\
\text { материалов, комплектующих, энергоноси- } \\
\text { телей, нарушение договоров транспортной } \\
\text { обеспеченности и т. д. }\end{array}$ & 0,37 & 120 & 400 \\
\hline 4 & Недобросовестность партнеров по проектам & 0,35 & 150 & 500 \\
\hline 5 & Срыв плановых показателей по доходам & 0,26 & 80 & 300 \\
\hline
\end{tabular}

\section{Таблица 2}

Снижение вероятности риска

\begin{tabular}{|c|c|c|c|c|c|}
\hline № метода & 1 & 2 & 3 & 4 & 5 \\
\hline 1 & 0,3 & 0,35 & 0,5 & 0,3 & 0,4 \\
\hline 2 & 0,4 & 0,55 & 0,25 & 0,4 & 0,35 \\
\hline 3 & 0,3 & 0,35 & 0,25 & 0,3 & 0,15 \\
\hline 4 & 0,3 & 0,3 & 0,4 & 0,15 & 0,25 \\
\hline 5 & 0,3 & 0,1 & 0,2 & 0,3 & 0,2 \\
\hline
\end{tabular}

Таблица 3

Время, необходимое для реализачии метода воздействия на риск

\begin{tabular}{|c|c|c|c|c|c|}
\hline № метода & 1 & 2 & 3 & 4 & 5 \\
\hline 1 & 100 & 220 & 180 & 140 & 180 \\
\hline 2 & 140 & 100 & 110 & 250 & 150 \\
\hline 3 & 100 & 100 & 150 & 80 & 80 \\
\hline 4 & 200 & 120 & 100 & 100 & 130 \\
\hline 5 & 70 & 50 & 50 & 70 & 100 \\
\hline
\end{tabular}

Таблица 4

Издержки, связанные с реализаиией метода воздействия на риск

\begin{tabular}{|c|c|c|c|c|c|}
\hline № метода & 1 & 2 & 3 & 4 & 5 \\
\hline № события & 250 & 500 & 800 & 500 & 400 \\
\hline 1 & 250 & 150 & 170 & 400 & 300 \\
\hline 2 & 350 & 500 & 350 & 250 & 250 \\
\hline 3 & 400 & 250 & 350 & 300 & 400 \\
\hline 4 & 150 & 180 & 200 & 250 & 400 \\
\hline 5 & \multicolumn{4}{|c}{} \\
\hline
\end{tabular}

$$
\mu_{i j}^{k}=1-\frac{q_{i j}^{k}-\underline{Q^{k}}}{\overline{Q^{k}}-\underline{Q^{k}}},
$$

В условиях рассматриваемого примера возникает необходимость использования обратной формулы, т. к. в соответствии с принципом выбранных нами критерии эффективности тем лучше, чем ниже их показатели.

Расчетные характеристики приведены в табл. 5-7:

Таблица 5

Частные оценки качества по критерию «Снижение вероятности риска»

\begin{tabular}{|c|c|c|c|c|c|}
\hline № метода & 1 & 2 & 3 & 4 & 5 \\
\hline 1 & 0,56 & 0,44 & 0,11 & 0,56 & 0,33 \\
\hline 2 & 0,33 & 0,00 & 0,67 & 0,33 & 0,44 \\
\hline 3 & 0,56 & 0,44 & 0,67 & 0,56 & 0,89 \\
\hline 4 & 0,56 & 0,56 & 0,33 & 0,89 & 0,67 \\
\hline 5 & 0,56 & 1,00 & 0,78 & 0,56 & 0,78 \\
\hline
\end{tabular}

Таблица 6

Частные оценки качества по критерию «время реализации»

\begin{tabular}{|c|c|c|c|c|c|}
\hline № метода & 1 & 2 & 3 & 4 & 5 \\
\hline 1 & 0,75 & 0,15 & 0,35 & 0,55 & 0,35 \\
\hline 2 & 0,55 & 0,75 & 0,70 & 0,00 & 0,50 \\
\hline 3 & 0,75 & 0,75 & 0,50 & 0,85 & 0,85 \\
\hline 4 & 0,25 & 0,65 & 0,75 & 0,75 & 0,60 \\
\hline 5 & 0,90 & 1,00 & 1,00 & 0,90 & 0,75 \\
\hline
\end{tabular}


Таблица 7

Частные оценки качества по критерию «стоимость реализации»

\begin{tabular}{|c|c|c|c|c|c|}
\hline № метода & 1 & 2 & 3 & 4 & 5 \\
\hline 1 & 0,85 & 0,46 & 0,00 & 0,46 & 0,62 \\
\hline 2 & 0,85 & 1,00 & 0,97 & 0,62 & 0,77 \\
\hline 3 & 0,69 & 0,46 & 0,69 & 0,85 & 0,85 \\
\hline 4 & 0,62 & 0,85 & 0,69 & 0,77 & 0,62 \\
\hline 5 & 1,00 & 0,95 & 0,92 & 0,85 & 0,62 \\
\hline
\end{tabular}

Необходимо вычислить оценки требования к качеству:

$$
\varepsilon_{j}^{k}=1-\frac{Q m_{j}^{k}-\underline{Q^{k}}}{\overline{Q^{k}}-\underline{Q^{k}}} .
$$

Матрица этих характеристик приведена в табл. 8.

Таблица 8

Требования к качеству методов

\begin{tabular}{|c|c|c|c|}
\hline № критерия & 1 & 2 & 3 \\
\hline 1 & 0,16 & 0,12 & 0,31 \\
\hline 2 & 0,29 & 0,24 & 0,77 \\
\hline 3 & 0,40 & 0,59 & 0,62 \\
\hline 4 & 0,44 & 0,41 & 0,46 \\
\hline 5 & 0,64 & 0,82 & 0,77 \\
\hline
\end{tabular}

Следующим шагом определим матрицу интегральных показателей трудности устранения рисков по формулам:

$$
\begin{gathered}
d_{i j}^{k}=\frac{\varepsilon_{j}^{k}\left(1-\mu_{i j}^{k}\right)}{\mu_{i j}^{k}\left(1-\varepsilon_{j}^{k}\right)} ; \\
S d_{i j}=\sum_{k=1}^{l} v^{k} d_{i j}^{k}=1-\prod_{k=1}^{l}\left(1-d_{i j}^{k}\right)^{v^{k}} .
\end{gathered}
$$

Данные представлены в табл. 9, все значения выше 1 заменены на 1.

Трудность применения методов

\begin{tabular}{|c|c|c|c|c|c|}
\hline № метода & 1 & 2 & 3 & 4 & 5 \\
\hline 1 & 0,10 & 0,49 & 1,00 & 0,25 & 0,32 \\
\hline 2 & 0,69 & 1,00 & 0,16 & 1,00 & 0,84 \\
\hline 3 & 0,57 & 1,00 & 1,00 & 0,41 & 0,18 \\
\hline 4 & 1,00 & 0,48 & 1,00 & 0,18 & 0,45 \\
\hline 5 & 1,00 & 0,05 & 0,36 & 1,00 & 1,00 \\
\hline
\end{tabular}

$$
=\ln \left(\frac{1}{1-S d_{i j}}\right) \text {, поставив прочерк в ячейках, }
$$

где трудность равна единице, т. к. оценка в этой ячейке не приемлема.

Таблица 10

Логарифмические оценки трудности

\begin{tabular}{|c|c|c|c|c|c|}
\hline № метода & 1 & 2 & 3 & 4 & 5 \\
\hline 1 & 0,11 & 0,67 & - & 0,29 & 0,38 \\
\hline 2 & 1,16 & - & 0,17 & - & 1,81 \\
\hline 3 & 0,85 & - & - & 0,52 & 0,20 \\
\hline 4 & - & 0,65 & - & 0,19 & 0,59 \\
\hline 5 & - & 0,05 & 0,44 & - & - \\
\hline
\end{tabular}

Применив венгерский метод решения задачи о назначениях, используя в качестве исходных данных данные табл. 10, получим итоговое распределение методов и рисковых событий (табл. 11).

Таблица 11

Соответствие рисковых событий и методов

\begin{tabular}{|c|c|c|c|c|c|}
\hline № метода & 1 & 2 & 3 & 4 & 5 \\
\hline 1 & 1 & 0 & 0 & 0 & 0 \\
\hline 2 & 0 & 0 & 1 & 0 & 0 \\
\hline 3 & 0 & 0 & 0 & 0 & 1 \\
\hline 4 & 0 & 0 & 0 & 1 & 0 \\
\hline 5 & 0 & 1 & 0 & 0 & 0 \\
\hline
\end{tabular}

Вероятностная оценка эффективности устранения рисков нарушения эффективности системы будет иметь вид:

$$
p=\sum_{i=1}^{n} \sum_{j=1}^{m} S d_{i j} x_{i j}=0,52
$$

Полученная оценка указывает на ожидаемую вероятность нарушения функционирования системы управления предприятием в случае, если будет иметь место хотя бы одно рисковое событие, описанное выше [11].

\section{ЗАКЛЮЧЕНИЕ}

В статье сформулирована модель оценки расчетно-аналитических риск-показателей, позволяющая учитывать многокритериальность оценок внешних и внутренних факторов риска, основанная на оценках типа трудности достижения цели. Рассмотрен механизм оценки минимизации трудности устранения рисков. Проведенный экспериментальный расчет позволил определить ком- 
плексные риск-показатели нарушения функционирования системы и найти оптимальное распределение методов устранения рисков по рисковым событиям. Возможность вероятностной трактовки оценок типа трудности достижения цели как риска недостижения цели функционирования системы позволяет использовать эти оценки как специальные многокритериальные риск-показатели в различных по своему типу системах.

\section{СПИСОК ЛИТЕРАТУРЫ}

1. Елисеева, Т. А. Снижение риска производителя технических систем на этапе проектирования совершенствованием оценки надежности: дис. ... канд. техн. наук : 05.02.23 / Т. А. Елисеева. - Тула, 2017. - 139 с.

2. Ажмухамедов, И. М. Системный анализ и управление социотехническими системами комплексного обеспечения информационной безопасности / И. М. Ажмухамедов // Проблемы информационной безопасности. Компьютерные системы. - 2013. - № 1. - С. 132-151.

3. Браун, А. А. Методы оценки рисков в системе управления безопасностью труда / А. А. Браун // Gaudeamus Igitur. - № 4. - 2015.

4. Родионова, М. А. Анализ рисков на производственном предприятии / М. А. Родионова, Л. А. Редько // Gaudeamus Igitur. - № 4. 2015.

5. Руссман, И. Б. Системный анализ социально-экономических процессов / И. Б. Руссман, Д. С. Чембарцев // Вестник ВГУ. - 2006. № 2. - С.163-165.

6. Афанасьев, М. Ю. Исследование операций в экономике: модели, задачи, решения. Учеб. пособие / М. Ю. Афанасьев, Б. П. Суворов. - М. : ИНФРА-М, 2003. - 444 с. (Серия «Высшее образование»).

7. Алексеев, А. П. О вероятностной оценке качества достижения цели системы / А. П. Алексеев // Наука и образование в XXI веке: сборник трудов по материалам международной научно-практической конференции, г. Тамбов 28 февраля 2017 г. - Тамбов : ООО «Консалтинговая компания Юком», 2017. - № 2-2(18). - C. 11-13.
8. Алексеев, А. П. О комплексной оценке качества функционирования системы / А. П. Алексеев // Академическая наука - проблемы и достижения: материалы XII международной научно-практической конференции, 15-16 мая 2017 г. - CreateSpace, 4900 LaCross Road, North Charleston, SC, USA. - 2017.

9. Каплинский, А. И. Имитационное моделирование стимулирующего развития источников ресурсов в мелиорации / А. И. Каплинский, И. Б. Руссман, В. М. Умывакин // Математическое моделирование в проблемах рационального природопользования: Тез. докл. Обл. Х школы-семинара. - Ростов н/Д., 1986. - С. 220-221.

10. Алексеев, А. П. Использование трудности достижения цели для оценки синергетического эффекта при создании интегрированных структур / А. П. Алексеев, Г. В. Абрамов, И. Н. Булгакова // Международный научно-исследовательский журнал, 2015. - № 10-2 (41). - C. 6-10.

11. Алексеев, А. П. Формирование оптимальной структуры системы с учетом ее функциональной эффективности / А. П. Алексеев, Г. В. Абрамов, И. Н. Булгакова // Международный научно-исследовательский журнал, 2016. - № 8-3 (50). - C.12-20. 
Алексеев Антон Павлович - аспирант кафедры математического и прикладного анализа, Воронежский государственный университет. Email: evil-emperor@mail.ru

Абрамов Геннадий Владимирович - д-р техн. наук, профессор, зав. кафедрой математического обеспечения ЭВМ факультета ПММ Воронежского государственного университета.

Email: abramov_g@amm.vsu.ru

Булгакова Ирина Николаевна - д-р экон. наук, кафедра математических методов исследования операций факультета ПММ Воронежского государственного университета. Email: bulgakova-i-n@amm.vsu.ru
Alekseev Anton Pavlovich - Postgraduate student of the Department of Mathematical and Applied Analysis, Voronezh State University. Email: evil-emperor@mail.ru

Abramov Gennady Vladimirovich - Prof, Doctor of Technical Sciences, Chief of Chair of Mathematical Support of Information Systems, Voronezh State University.

E-mail: abramov_g@amm.vsu.ru

Bulgakova Irina Nikolaevna - Doctor of Economics, Mathematical Methods of Operations Research, Voronezh State University.

E-mail: bulgakova-i-n@amm.vsu.ru 